La revue La revue pour l'histoire du CNRS

POUR L'HISTOIRE DU CNRS

$22 \mid 2008$

La géopolitique mode d'emploi

\title{
Le partage des savoirs scientifiques
}

Les centres de culture scientifique, technique et industrielle

Étienne Guyon et Bernard Maitte

\section{(2) OpenEdition}

Journals

Édition électronique

URL : https://journals.openedition.org/histoire-cnrs/8322

DOI : $10.4000 /$ histoire-cnrs.8322

ISSN : 1955-2408

Éditeur

CNRS Éditions

Édition imprimée

Date de publication : 3 octobre 2008

ISBN : 978-2-271-06676-3

ISSN : $1298-9800$

Référence électronique

Étienne Guyon et Bernard Maitte, "Le partage des savoirs scientifiques », La revue pour I'histoire du CNRS [En ligne], 22 | 2008, mis en ligne le 03 octobre 2010, consulté le 20 mai 2021. URL : http:// journals.openedition.org/histoire-cnrs/8322 ; DOI : https://doi.org/10.4000/histoire-cnrs.8322

Ce document a été généré automatiquement le 20 mai 2021

Comité pour l'histoire du CNRS 


\title{
Le partage des savoirs scientifiques
}

\author{
Les centres de culture scientifique, technique et industrielle
}

\author{
Étienne Guyon et Bernard Maitte
}

Les conditions de la créationAvant la naissance des CCST

1 Les actions de popularisation d'une science en mouvement, en particulier celles tournées vers les jeunes, sont bien antérieures aux créations des premiers CCST, qui se sont inspirés de ces exemples. Les animations menées par le Palais de la Découverte illustrent cette démarche. Le Palais entretenait des relations continues avec le monde de la recherche ainsi que l'avait souhaité Jean Perrin à sa création en 1937, qui fut synchrone avec celle de ce qui allait devenir très vite le CNRS. Dans les années 1960, le Palais créait des expositions et en assurait leur itinérance. Les échanges d'expositions se faisaient alors dans l'esprit d'un "troc», bien différent d'une démarche plus courante aujourd'hui de vente ou location, et ils conduisaient à la constitution d'un réseau entre animateurs culturels scientifiques. Par ailleurs, les clubs Jean-Perrin d'animation pour des jeunes se créent en 1962 à l'intérieur du Palais (chimie, physique, électronique, science du vivant et de la Terre) avant de conduire à des stages de vacances en particulier d'astronomie. D'autres associations se mettent alors en place, telles que l'ANSTJ (qui deviendra Planète Sciences) en 2002 (avec l'aide du CNES à partir des projets de lancements de micro fusées et de ballons) et les clubs santé avec l'Inserm. Des clubs d'amateurs prospèrent aussi autour des Muséums (National ou de province), ainsi que du musée du Cnam. L'association nationale des clubs scientifiques créée en 1962, qui organisera des temps de rencontre avec les exposciences permet de fédérer les activités diverses de ces clubs ${ }^{1}$.

Le démarrage à Grenoble

2 En 1966, à l'occasion de la préfiguration d'une Maison de la culture à Grenoble, un groupe de scientifiques défend l'idée d'y intégrer une animation scientifique, au même titre que les autres activités, musicales, théâtrales, cinématographiques... Son but est de «faire admettre que les sciences sont un élément constitutif de la culture contemporaine ». Deux ans plus tard, à l'ouverture de cette maison de la culture, un secteur «sciences» est mis en place, qui veut suivre le modèle de présentation du Palais de la Découverte (expositions, animations, conférences). Le succès rencontré 
(avec un public aussi important pour le secteur «sciences" que pour les autres activités), la volonté de mettre en itinérance les expositions, des difficultés aussi de relations avec ces autres activités, font qu'une volonté de créer un «Centre culturel scientifique » indépendant se manifeste en 1974. Un colloque se tient cette même année avec les animateurs de la physique dans la rue sur la place des sciences dans l'action culturelle, qui conclut que l'on ne peut en rester à présenter des explications sur la science, mais qu'il faut "parler des applications et des implications ». En 1975, une association de type loi de 1901 est créée, qui bénéficie de soutiens de la ville, du ministère de la Culture, de la DGRST. Avec le bénévolat de nombreux scientifiques, des expositions itinérantes sont produites, des «Camps scientifiques » organisés. En 1979, la ville décide de mettre à disposition des locaux, La Casemate, pour un centre culturel scientifique et technique. Une équipe se met en place, des statuts sont votés. C'est le démarrage du premier CCST. Son équipe contribuera fortement à l'encadrement de la première équipe de la Cité des sciences de la Villette après 1982.

D'autres actions : la physique dans la rue et le GLACS

3 Au mois de septembre 1973, à l'occasion d'un congrès international de physique des particules, Michel Crozon, avec un congressiste suisse, Michel Sonderegger, décide de conduire une animation de physique dans la rue qui présente des expériences de physique générale ainsi que de physique nucléaire, comme une grande chambre à étincelles qui visualise l'arrivée aléatoire de rayons cosmiques. Ils sont aidés par le directeur du relais culturel d'Aix, Charles Nugues, qui menait annuellement des actions de musique dans la rue. Cette animation connaîtra un grand succès. Un des auteurs de cet article (É. Guyon) était par hasard en vacances à Aix à cette date avec de nombreux enfants qui se passionnent pour ces rencontres autour d'expériences... Deux ans plus tard, à l'occasion du congrès national de la société française de physique à Dijon, on lui demande de mettre en place une animation en parallèle avec le Congrès. Comment refuser! L'animation, autour d'un thème général la Terre et ses énergies sera de nouveau un succès. Le pli était pris et de nombreux congrès seront accompagnés de manifestations dans la ville. Ainsi, à Poitiers en 1977, la manifestation prend une ampleur particulière et associe une quarantaine d'associations, l'École des beaux-arts, de nombreux universitaires. Une trentaine d'animations, trois journées Physique dans la rue, onze expositions itinérantes, 22 conférences-débats, une exposition sont réalisées. Après cette manifestation, un groupe informel se constitue. Il organise en 1978 des animations et des expositions itinérantes en milieu rural "énergies nouvelles », «les oiseaux de chez nous », en 1979 «la science et l'enfant », « la fête du soleil », en 1980 "Pop santé ». Le groupe forme alors le projet de créer une Maison des sciences, dont le but est d'instaurer un dialogue art/sciences et de mettre en relation les domaines des sciences, de l'industrie, de l'artisanat, des cultures techniques et artistiques. Il conduira à la réalisation de l'espace Mendès-France, toujours bien vivant.

Cette démarche reposait sur la juste prise de conscience par les chercheurs qu'il fallait informer (et écouter!) le public, en particulier celui qui ne va pas dans les musées, et sur le besoin de se justifier sur la légitimité d'une recherche scientifique non finalisée (les manifestations impliquaient presque exclusivement des chercheurs de sciences de base). On peut penser que ces actions vont inspirer les opérations de Science en fête lancées en 1992. La manifestation d'Aix marque aussi le début d'un mouvement de réflexion et d'action qui a impliqué des scientifiques, des curieux et des sociologues. Ce mouvement fut initié par le colloque de 1974 de Grenoble ; il fut alors suggéré de créer une structure permanente d'accompagnement d'actions culturelles en science, baptisée 
GLACS (Groupe de liaison pour une action culturelle scientifique). Elle eut pour premier président Michel Crozon. Il accompagnera cette association de façon continue avec clairvoyance et bon sens. La cheville ouvrière en était Marie-Simone Detoeuf, qui a profité du soutien logistique de l'IN2P3 où elle travaillait et qui a suivi de bout en bout ce groupe de liaison tant sur le plan de la logistique que sur le plan de l'animation, jusqu'à son décès le jour de Pâques 2007, quelques mois avant Michel.

5 L'action sera marquée par un questionnement et une mise en cause constante, par les non scientifiques censés représenter un public intéressé mais ignorant, et des scientifiques. É. Guyon y participera ; cette réflexion est à mettre en compte dans le bagage qu'il emportera lorsqu'il présidera le comité d'orientation de la Villette à la période de son ouverture ou au Palais de la Découverte qu'il dirigera à partir de 1988. Marie Simone nous a laissé en héritage un site remarquable ${ }^{2}$ qui permet de suivre l'action du GLACS sur plus de 20 ans à travers ses réunions mensuelles, ses animations, colloques et ses réalisations variées, et aussi de connaître l'évolution des manifestations de culture scientifique dans cette période.

6 Nous retiendrons dans ce très riche ensemble de documents deux actions. La première est la création d'une exposition La danse de l'Univers, qui accompagne la présentation de propriétés fondamentales de la physique des particules par des œuvres d'art qui rendent lisible ce qui en fait ne l'est pas. La seconde est, dans l'esprit des cabinets du XVIII ${ }^{e}$ siècle, le salon de Madame du Deffant high tech, qui réunit pendant deux ans une trentaine de personnes d'horizons divers, "gens de lettres et gens de sciences, ignorants et savants, [...], cartésiens et baladins, croyants et mécréants [...], en quête de repères pour notre temps", autour de trois thèmes: la mécanique quantique, la représentation de l'Univers, la biologie (vie et mort).

Les Boutiques de sciences

7 Le panorama des actions menées par les scientifiques ne serait pas complet si nous ne mentionnions la création dans les années 1980 des Boutiques des sciences, à l'image de celles qui fonctionnaient aux Pays-Bas depuis les années 1970. Ces boutiques voulaient répondre à des besoins sociaux en mettant en contact direct des scientifiques et des personnes confrontées à des problèmes de recherche. Lieux de médiation, ces structures recevaient des demandes concrètes de la société civile (associations, entreprises), les formulaient en langage scientifique, trouvaient les interlocuteurs compétents pour y répondre ou pour mener les recherches nécessaires. Des boutiques s'ouvrirent à Grenoble, Lyon, Paris, Strasbourg mais ne trouvèrent pas d'écho autre qu'universitaire avant 1981, date à partir de laquelle s'ouvrirent aussi celles de Lille, Marseille et Orsay. Par la suite, les boutiques s'adosseront ou non aux CCST, mais elles disparaîtront peu à peu à partir de 1985. La dernière (Strasbourg) fermera en 1991... Toutefois, la Fondation science citoyenne prévoyait d'en rouvrir une à l'ENS Cachan en 2008 !

Le développement des CCSTLes assises de la recherche et de la technologie

Ce mouvement sera accompagné d'initiatives de l'État: la création en 1972 du Bureau national d'information scientifique et technique (BNIST); le lancement en 1979 par Valéry Giscard d'Estaing du projet de La Villette reprenant l'idée des Science Centers américains et permettant - grâce au prestige de la science - de sortir de façon honorable des scandales immobiliers sur le site ; la transformation, cette même année, du BNIST en mission interministérielle (Midist)... Mais c'est l'arrivée de la gauche au pouvoir en 1981 qui va donner l'occasion au mouvement de s'épanouir, de se structurer 
et de s'exprimer politiquement. Tout semble alors possible, et les initiatives fleurissent, tant militantes qu'institutionnelles.

C'est d'abord le ministère de la Culture qui crée en son sein une direction nouvelle, la DDC (Direction du développement culturel), d'où partent les initiatives nouvelles de ce ministère (telles que la Fête de la musique et le soutien aux actions culturelles scientifiques); c'est le ministre d'État Jean-Pierre Chevènement qui dynamise la Midist, la dote de moyens, organise les Assises de la recherche et de la technologie. Les assises régionales sont un temps où les militants du partage du savoir peuvent se regrouper, trouver l'écho nécessaire, formuler de nouveaux projets, à l'image de celui que forme à Nice le physicien Jean-Marc Lévy-Leblond qui fonde l'Anais et théorise le mouvement des CCST. Ceux-ci devront "mettre la science en culture", de manière à "atteindre des objectifs économiques, politiques et sociaux, culturels et éducatifs, intellectuels et scientifiques fondamentaux». Ils offriront divers espaces spécialisés (expositions, exploratoire, documentation, salles d'actualités, lieux de débats et de confrontations, planétarium...) afin de s'adapter à la diversité des motivations des citoyens. Les phrases utilisées seront reprises dans de multiples documents. En Seine-Saint-Denis, la Fondation 93 veut privilégier la mise en réseau des structures culturelles du département par la mise en circulation d'expositions et de "valises expositions", et réaliser un "CCST sans murs »; à Lille est créée, par un des auteurs de cet article (B. Maitte) et un groupe de scientifiques, d'enseignants du secondaire, d'ingénieurs et de syndicalistes, l'Alias, qui veut mettre en place un CCST réunissant les caractéristiques des deux projets qui viennent d'être cités ainsi qu'une Boutique des sciences.

D'autres centres sont envisagés à Marseille, Poitiers, Rennes... Les Assises nationales de la recherche et de la technologie vont assurer le débouché politique indispensable au mouvement. Elles conduisent à la loi d'orientation et de programmation de la recherche (1982) et à ses programmes mobilisateurs (dont le $n^{\circ} 6$, "Promotion du français, langue scientifique, et diffusion de la culture scientifique et technique »), qui assignent aux enseignants-chercheurs trois missions : la recherche, l'enseignement et la mise à disposition sociale de leurs travaux. C'est sur ces dispositions que vont s'appuyer un peu partout en France les militants pour développer leurs actions. En 1983, le IX $\mathrm{X}^{\mathrm{e}}$ plan, dans son programme prioritaire d'exécution $\mathrm{n}^{\circ} 3$, indique dans le paragraphe Promouvoir la culture et l'information scientifique et technique: "Un réseau de centres de culture scientifique et technique sera mis en place dans les régions, articulé avec le musée de La Villette». C'est alors que la Midist, sous la pression de mouvements de gauche ajoute le « $\mathrm{i}$ » de « industriel » aux CCST et que le ministère de la Culture crée le "Conseil national de la culture scientifique, technique et industrielle». Mais cette adjonction ne sera pas prise souvent en compte dans la réalité.

La création des CCSTI

11 Dès lors, les propositions des initiateurs vont être soutenus par les ministères. La Midist et la DDC, organismes non décentralisés, donnent directement des moyens à celles des associations qui trouvent un montant égal de subventions à l'échelle locale. Des départements (Seine-Saint-Denis) ou des villes (Grenoble, Poitiers, Rennes...) abondent les financements. C'est plus difficile ailleurs; mais l'action d'un DRRT, et l'opportunité de la mise en place des contrats de plan État/Régions, permettent de lancer des opérations significatives.

12 Nous prendrons comme exemple de ce développement l'Alias. L'association obtient l'appui de l'État, puis de la région Nord-Pas de Calais. Elle développe alors des actions 
destinées à préfigurer et évaluer les diverses fonctions que devra remplir le CCST. Elle réalise des expositions, toutes itinérantes. Des valises-explorations, inspirées à la fois de la Fondation 93, des expositions à tiroirs du Bauhaus et des manipulations des cabinets de curiosités, circulent entre différentes structures éducatives de la région et sont transportées dans des coffres de voitures. Des émissions radiophoniques sont programmées. Des salles d'actualités (expositions et conférences-débats) soulèvent des questions vives posées par l'introduction des technologies dans la société. Un centre de documentation fournit des malles bibliographiques itinérantes et mène des opérations sur le livre scientifique de jeunesse Livre ta science. Des espaces enfants sont réalisés dans des écoles. Un planétarium gonflable est installé jusque dans les stations de métro de Lille. Toutes ces actions soutiennent l'idée de construire un CCST, permettent de réfléchir à ce que seront ses espaces, ses fonctions, son rayonnement hors des murs, testent avec les différents partenaires ce que seront les réseaux culturels scientifiques.

Ces initiatives permettent d'offrir le moyen de mettre en œuvre une politique qui demande «autant d'audace, une mobilisation comparable à celles déployées à une autre époque par Jules Ferry ". Elles sont menées dans des locaux de plus en plus importants, où sont réalisées en vraie grandeur chacun des futurs espaces de ce qui deviendra le forum des sciences.

14 Tous les porteurs de projets apprennent à se connaître et à coordonner leurs actions grâce à l'AMCSTI (fondée en 1982 sous la présidence de Hubert Curien) : elle fédère tous les musées et structures culturelles scientifiques. En 1985, un rapport sur les centres de culture scientifique, technique et industrielle (B. M.) contribue à donner une meilleure visibilité aux actions entreprises en Bretagne (Abret), à Rennes, Grenoble, Lille, Marseille, Nice, Poitiers, Seine-Saint-Denis (Fondation 93), Strasbourg et Toulouse. Il reste que toujours et partout des difficultés se manifestent quant à la perception de ce qu'est «la culture scientifique «: se réduit-elle à la diffusion des résultats de la recherche, à la vulgarisation scientifique, à l'animation de débats par les chercheurs ? "Comment peut-on parler d'une culture scientifique qui existerait actuellement et qu'il suffirait de diffuser, alors que la majorité des scientifiques détient un savoir hautement spécialisé et ignore le plus souvent le développement historique dans lequel se situe leur propre travail ?... Mettre la science en culture est une exigence... qui dépasse le cadre de ce qui a été vu jusqu'à présent et qui nécessite, pour être réalisée que soient créés des outils nouveaux, des lieux d'échanges et de rencontres : les CCSTI ", peut-on lire dans un rapport de l'Alias.

L'âge mûr

Tout ce mouvement s'épanouit sous l'influence de Hubert Curien², ministre de la Recherche, à partir de 1984. La Cité des sciences et de l'industrie ouvre ses portes en 1986. La rénovation des grands établissements parisiens (Muséum national, musée des Arts et Métiers, Palais de la Découverte), ainsi que de quelques musées de province est lancée après le rapport Héritier Augé4. Le réseau des CCSTI est soutenu et étendu : le ministère souhaite que s'ouvre au moins un CCSTI par région, ce qui ne sera pas tout à fait réalisé, pas plus que l'harmonisation du maillage de CCSTI avec la politique régionale de La Villette, vécue comme hégémonique. Des actions avec les grands organismes de recherche (mille chercheurs/ mille classes avec le CNRS, clubs de l'Inserm...) se développent. Grâce au Cirasti créé en 1984, le mouvement des exposciences est dynamisé. Des tentatives sont faites pour introduire des émissions scientifiques à la télévision... Cet élan continuera malgré les changements politiques, et sera réaffirmé à l'occasion des « États généraux de la CSTI » de 1989. En 1992, est lancée 
par Hubert Curien, à nouveau ministre, La Science en Fête à l'occasion du dixième anniversaire de l'implantation du ministère sur le site Descartes ${ }^{5}$.

Au-delà des fluctuations de l'intérêt des ministres qui lui ont succédé (un d'entre eux trouvait même que parler de "fête" à propos de la science était incongru!), le mouvement a continué jusqu'à ce jour avec des soutiens des différentes communautés territoriales. Dans le même temps, plusieurs CCSTI en dur se créent à Poitiers (1989), dans le Nord-Pas de Calais (Forum des sciences, 1996) qui prolongent leurs actions hors les murs; dans d'autres cas, ils restent des centres de création et de diffusion d'expositions, aux niveaux départemental (Fondation 93), régional (Abret) ou national et international (Centre sciences à Orléans).

Mis en place dans un temps bref, le dispositif français de ces CST est important ${ }^{6}$. Il présente un certain nombre de faiblesses: tutelles diverses où l'Éducation nationale n'est pas le partenaire majeur que l'on aurait attendu; querelles de territoires et de responsabilités; non uniformisation des carrières et impossibilité d'évolution professionnelle; disparité Paris/régions; financements de fonctionnement non assurés. Actuellement, nous pouvons dire qu'il n'y a quasi plus de politique nationale d'accompagnement de projets et de réalisation. De nouveaux établissements, créés avec des investissements assurés par des communautés locales, s'ouvrent à Bordeaux, Rennes et Lyon... Mais d'autres, malgré leur rayonnement, sont contraints de fermer faute de ce soutien (très récemment, à Marseille... deuxième ville de France !). Cet état de jachère est grandement préjudiciable à notre pays. Il est nécessaire qu'une réflexion ait lieu quant aux objectifs (pédagogiques, informative, culturelle, critique, publicitaire...), plus ou moins compatibles entre eux, des établissements créés et de leur avenir, réflexion que visait l'opportune création de la « Réunion nationale des CCSTI ", mais pour quels résultats?

Les CCST ont été créés pour aider les citoyens à mieux connaître et évaluer les enjeux de la société en proposant une information de base et un cadre de dialogue autour des sciences. Mais dans le même temps, ils se sont efforcés de présenter la science comme une partie intégrante de la culture ${ }^{7}$. La société qui réussira cette intégration pourra évaluer de manière plus lucide les enjeux culturels, économiques, sociaux, éthiques des développements technoscientifiques. Ce défi passe-t-il par une extension de ce qui existe actuellement ou par des formes nouvelles tenant compte des modes nouveaux d'échanges et de communication? La réponse à cette interrogation reste à trouver. Remerciements

Nous remercions Michel Briantais, Michel Darche, Jean-Claude Guiraudon, Charles Penel et Brigitte Vogler pour leurs informations sur l'histoire du mouvement d'animation culturelle scientifique.

Cet article devait être écrit avec Michel Crozon. Le décès brutal de celui-ci nous prive d'un témoin, acteur majeur de ces actions. Physicien des hautes énergies actif et passionné, honnête homme dont la richesse intellectuelle n'avait d'égale que la modestie et l'humour malicieux, il fut un précurseur des actions de diffusion de la science vers un large public à partir d'initiatives visant à développer la citoyenneté au sujet des applications scientifiques. Michel Crozon fut 
directeur de l'information scientifique et technique au CNRS après 1992. Préalablement, il accompagna, comme délégué à l'information scientifique et technique du ministère de la Recherche, sous Hubert Curien, la mise en place des CCSTI, permit aux clubs scientifiques de se développer et de se fédérer au sein du Cirasti, participa activement aux actions tournées vers les jeunes, dont « Les Petits Débrouillards » dont il était encore le parrain à sa mort. L'action de Michel est représentative de tout ce mouvement au sein duquel la création des CCSTI prend sens et auquel nous voulons associer sa mémoire. Nous avons, dans cet article, choisi de mettre en perspective le développement des CCSTI au sein du foisonnement qui s'est produit en France de 1973 (première manifestation « Physique dans la rue ») à 1992 (première « Science en Fête »). Voir Crozon M., Maitte B. « La culture scientifique en France : institutions, enjeux ». Esprit, $\mathrm{n}^{\circ} 10$, oct. 2001, pp. 105- à 119.

\section{NOTES}

1. Pour un historique voir : http://www.planete-sciences.org/national/ quisommesnous-historique.html

2. http://www.glacs.com

3. Guyon É. «Hubert Curien et la science en fête ». In Hubert Curien. Pour une politique internationale de la science, presses de l'ENS, 1994, p. 53.

4. Les musées de l'Éducation nationale. Mission d'étude et de réflexion. Rapport au ministre d'État, ministre de l'Éducation nationale. La Documentation française, Collection des rapports officiels, 1991.

5. «Science et culture en Europe », Alliage, n¹6-17, été/automne 1993.

6. Voir le site de l'AMCSTI : http://www.amcsti.fr et aussi http://www.ccsti.fr/ index.php?option=com

7. «Médiation et culture scientifique, Alliage, n59, déc. 2006. 
INDEX

Mots-clés : CCSTI, centres de culture scientifique technique et industrielle, culture scientifique, Boutiques de science, La physique dans la rue, vulgarisation

\section{AUTEURS}

\section{ÉTIENNE GUYON}

Étienne Guyon est professeur émérite et travaille au laboratoire de physique et mécanique des milieux hétérogènes de l'École supérieure de physique et de chimie industrielles de la ville de Paris (UMR 7636).

\section{BERNARD MAITTE}

Bernard Maitte, professeur d'histoire des sciences et d'épistémologie, est membre de l'UMR « Savoirs, textes, langage ». 